\title{
Cobertura vacinal em crianças de 12 a 23 meses de idade: estudo exploratório tipo Survey
}

\author{
Vaccination coverage in children aged 12 to 23 months: a Survey study
}

\section{Cobertura vacunal en niños de 12 a 23 meses de edad: estudio exploratorio tipo Surrey}

\author{
Denise Romão Pereira', Thais Aidar de Freitas Mathias" \\ Dorotéia Fátima Pelissari de Paula Soares'I", Wladithe Organ de Carvalho'v
}

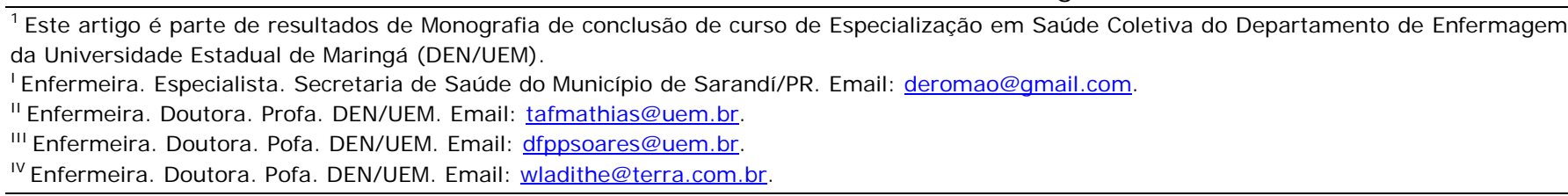

\section{RESUMO}

O objetivo deste estudo foi avaliar a cobertura vacinal de crianças de 12 a 23 meses de idade residentes no município se Sarandi, PR. Para tanto foi realizado inquérito domiciliar, de dezembro de 2003 a março de 2004 , para verificar as carteiras de vacinação das crianças residentes. A cobertura vacinal foi analisada segundo sexo, idade, tipo de vacina e município de realização da vacina. Das 740 carteiras de vacina avaliadas 77,6\% constava esquema vacinal completo, sem diferença quanto ao sexo. As coberturas mais altas ocorreram para a BCG de 99,6\%, para a vacina DTP/Tetra de 98\%, para a Pólio de 97,2\% e para a Hepatite B de 96,2\%. Somente $31,1 \%$ das crianças com 12 meses de idade apresentavam esquema vacinal completo. As vacinas BCG e DTP/Tetra tiveram maior taxa de evasão, 13,9\% e 11\%, respectivamente. Entre as estratégias para melhorar as taxas de cobertura vacinal e realização das vacinas nos intervalos preconizados pelo Ministério da Saúde, destacam-se a capacitação da equipe de enfermagem, do aprimoramento do acesso e da qualidade do atendimento nos serviços de saúde e nas salas de vacina.

Descritores: Imunização; Inquéritos domiciliares de saúde; Cobertura vacinal; Enfermagem; Saúde da criança.

\section{ABSTRACT}

The objective of this study was to evaluate the immunization coverage in children from 12 to 23 months of age resident in the municipality of Sarandi/PR-Brazil. For that a household interview survey was carried out to verify the vaccine cards of the resident children. Home visits were accomplished from December of 2003 to March of 2004. The vaccine coverage was analyzed according to gender, age, vaccine type and city of vaccination. Of the 740 vaccine cards assessed, $77.6 \%$ had the complete vaccine doses, with no difference regarding gender. The highest coverage was $99.6 \%$ for BCG, $98 \%$ for DTP/Tetra, $97.2 \%$ for Polio, $96.2 \%$ for Hepatitis B. Only $31.1 \%$ of children aged 12 months old had the complete vaccine coverage. The BCG and DTP/Tetra vaccines had the greatest rate of evasion, $13.9 \%$ and $11 \%$, respectively. Among the strategies to improve the vaccine coverage rates and the taking of vaccines in the standardized dates established by the Health department, it is worth mentioning the improvement of the access and the quality of the attendance in the vaccine rooms.

Descriptors: Immunization; Household interview surveys; Immunization coverage; Nursing; Child health

\section{RESUMEN}

Para evaluar la cobertura vacunal fue realizado averiguación de los carnés de niños de 12 a 23 meses residentes en el municipio de Sarandi - PR, de diciembre de 2003 a marzo de 2004. La cobertura vacunal fue analizada segundo sexo, edad, tipo de vacuna y municipio de realización. De los 740 carnés de vacuna evaluados 77,6\% constaban esquema vacunal completo, sin diferencia con relación al sexo. Las coberturas más altas fueron de 99,6\% para la BCG, 98\% para DTP/Tetra, 97,2\% para la Polio, 96,2\% para la Hepatitis B. De los niños con 12 meses de edad solamente 31,1\% presentaban esquema vacunal completo. Las vacunas BCG y DTP/Tetra tuvieron mayor tasa de evasión, 13,9\% y 11\%, respectivamente. Entre las estrategias para mejorar las tasas de cobertura vacunal y realización de las vacunas en los intervalos estandarizados por el Ministerio de la Salud, se destacan el perfeccionamiento del acceso y de la calidad del atendimiento en las salas de vacuna.

Descriptores: Inmunización; Cobertura vacunal; Enfermería; Salud del niño. 


\section{NTRODUÇÃO}

Considerando a complexidade da situação das doenças infecciosas no Brasil, uma importante tendência é o decréscimo daquelas para as quais se dispõe de instrumentos eficazes de prevenção e controle como para as imunopreveníveis, que têm como exemplos recentes a eliminação da poliomielite e do sarampo, graças à manutenção de altas taxas de coberturas vacinais, principalmente entre os menores de um ano.

No Brasil, o Programa Ampliado de Imunização (PAI), criado em 1974 pela Organização Mundial da Saúde, foi regulamentado em 1975 quando foi instituído o Programa Nacional de Imunizações (PNI). O PNI é citado como referência mundial por sua excelência comprovada, já tendo organizado campanhas de vacinação no Timor Leste, cooperando com a Palestina, Cizjordania e Faixa de Gaza, além de estabelecer cooperação técnica com vários países como Estados Unidos, México, Guiana Francesa, Argentina, Paraguai, Uruguai, entre outros ${ }^{(1)}$.

O PNI é peça importante no controle das doenças transmissíveis, pois combina a vacinação de rotina, os dias nacionais de vacinação, as campanhas periódicas e a vigilância epidemiológica ${ }^{(2)}$. Em 1984 houve a primeira tentativa em utilizar os dias nacionais de vacinação contra a Poliomielite para aplicar as outras vacinas do calendário, vacina tríplice e contra o sarampo. Essa experiência foi retomada em 1990 com excelentes resultados contribuindo para recuperar os índices de cobertura vacinal para as outras vacinas ${ }^{(1)}$.

Uma razão que torna a vacinação um método altamente viável é a de possuir o melhor resultado em termos custo-benefício, visto que o gasto com vacinação é muito inferior ao gasto com pessoas acometidas por tais doenças que necessitam de acompanhamento da equipe de saúde, medicação, internação, exames, além das seqüelas ou até mesmo a morte, situações de custo econômico e social inquestionáveis.

O Ministério da Saúde estabelece meta de cobertura vacinal de $90 \%$ para a vacina contra a tuberculose (BCG), 95\% para Poliomielite, 95\% para a vacina Tetravalente (contra tétano, difteria, coqueluche - DPT e meningite - Hib), 95\% para Hepatite B, 95\% para Vacina Tríplice Viral (VTV) (contra caxumba, rubéola e sarampo) e 100\% para Febre Amarela (FA) ${ }^{(2)}$.

A prática de vacinação tem conseguido reduzir o número de casos ou mesmo erradicar doenças. A poliomielite, por exemplo, foi considerada erradicada das Américas em 1994 pela Organização Mundial de Saúde ${ }^{(3)}$. Isto ocorreu no Brasil devido ao número crescente de doses aplicadas e pela aproximação dos resultados esperados de atingir a meta estabelecida pelo Ministério da Saúde. O alcance das metas nos dias nacionais de vacinação contra a poliomielite não deve diminuir o empenho à cobertura vacinal em vacinações de rotina, visto que sua redução possibilitaria a reintrodução da doença no país, pois em outras regiões do planeta o número de casos ainda é alto, como se verificou em 1997 com registro de 7.975 casos em todo o mundo ${ }^{(3)}$.

A falta de manutenção de alta taxa de cobertura da vacinação contra o sarampo em todos os municípios brasileiros possibilitou novos surtos no ano de 1997, como ocorreu no Estado de Santa Catarina onde foi observado que a cobertura vacinal foi menor do que os níveis necessários para a erradicação $(95 \%)$ e controle $(90 \%)^{(4)}$. No município de São Paulo a ocorrência da epidemia de sarampo em 1997 surpreendeu o programa de vigilância epidemiológica e serviu de alerta para a necessidade de pesquisas para conhecer a correta cobertura vacinal em menores de 1 ano $^{(5)}$ e ainda, chama a atenção para observar a qualidade de assistência à vacinação nas atividades de rotina nos serviços de saúde.

Por isso a avaliação da cobertura vacinal pode contribuir para obtenção de respostas relacionadas à efetividade da ação para detectar se a população infantil encontra-se imunizada, além da identificação de pontos frágeis das atividades de vacinação. O conhecimento preciso da cobertura vacinal é um dos elementos essenciais para a vigilância epidemiológica, pois permite verificar se as metas estabelecidas para cada vacina estão sendo alcançadas e mais ainda, acompanhar a existência de pessoas suscetíveis às doenças imunopreveníveis na população.

A cobertura vacinal pode ser feita por meio de dois métodos. O primeiro é o método administrativo, estimado rotineiramente a partir das informações dos boletins produzidos pelos serviços de saúde que são preenchidos pelas equipes de enfermagem nas salas de vacinação em Unidades Básicas de Saúde (UBS), ambulatórios e hospitais. Esse é o método mais utilizado para avaliar a cobertura vacinal devido à disponibilidade e facilidade de acesso aos dados. Entretanto, existe a possibilidade da cobertura vacinal assim avaliada não refletir a cobertura real, pois as informações são relativas às doses aplicadas, independentemente da idade e do intervalo entre as aplicações, acarretando o desconhecimento da permanência de indivíduos suscetíveis, além de que as doses aplicadas com intervalos mais curtos podem não desenvolver imunidade. Soma-se a isso que os dados de produção estão sujeitos a erros de registro (sub-registro, duplicidade de dados) e não mostram algumas especificidades como informações sobre aquelas crianças que, embora residindo na área de influência do serviço de saúde, foram vacinadas em serviços de outras áreas. 
O segundo método de análise da cobertura vacinal, chamado de método estatístico, é obtido por meio de inquéritos populacionais, apresenta vantagens, pois não sofre influência da invasão ou evasão de crianças já que os dados do numerador estão contidos no denominador. Destaca-se ainda que os inquéritos representam a única fonte de dados para obtenção de informações, tanto de usuários quanto de não usuários do SUS, dos que recebem os cuidados e dos que não recebem ou mesmo daqueles que não têm acesso ao sistema de saúde. Permitem também, quando programado, coletar as variáveis socioeconômicas da área de abrangência e das famílias, constatar a idade da administração de cada dose de vacina e o intervalo entre elas, conseguir informações sobre as crianças que, embora residindo na área de influência do serviço de saúde, foram vacinadas em outros serviços e as que receberam vacinas em serviços não vinculados ao Sistema Único de Saúde (SUS). A realização de inquérito permite conhecer a cobertura vacinal real, compará-la com a cobertura administrativa, identificar o acesso e a adesão ao programa de imunizações, bem como as desigualdades sociais existentes na cobertura vacinal $^{(6)}$. Além disso, torna possível conhecer a aceitabilidade dos serviços de imunização e a percepção dos usuários quanto à vacinação, compreender 0 contexto de baixas coberturas vacinais, as razões para a não vacinação ou vacinação incompleta, bem como subsidiar e avaliar estratégias locais de inclusão adotadas pelos programas de vacinação(7).

Tendo em vista a ocorrência de surtos de sarampo no Brasil e países da América Latina ${ }^{(4)}$ a possibilidade de reintrodução da coqueluche $^{(8)}$ e também todas as possibilidades de fragilidade na avaliação da cobertura vacinal realizada por meio dos dados de produção ${ }^{(7)}$, é importante que os inquéritos populacionais sejam feitos com maior freqüência para que se conheça a real cobertura vacinal de determinada localidade. É necessário manter a redução na incidência das doenças imunopreveníveis e atingir novas metas de seu controle ou eliminação, motivo pelo qual o fortalecimento da capacidade das ações de vigilância epidemiológica e o aumento da homogeneidade da cobertura vacinal de rotina terem sido definidos como estratégias pelo Ministério da Saúde ${ }^{(9)}$. Assim, foi objetivo deste estudo avaliar a cobertura vacinal de crianças de 12 a 23 meses de idade residentes no município de Sarandi, PR, por meio de inquérito domiciliar.

\section{MÉTODOS}

Este foi um estudo do tipo transversal, com dados provenientes de inquérito domiciliar vacinal para crianças de 12 a 23 meses de idade residentes no município de Sarandi, realizado no período de novembro de 2003 a março de 2004. Sarandi está localizada na região noroeste do Estado do Paraná a uma distância de $425 \mathrm{~km}$ de Curitiba fazendo limite com os municípios de Marialva e Maringá. A área entre esses municípios é mínima, tendo em alguns pontos apenas ruas como delimitação o que possibilita fácil acesso entre eles. Elevado à categoria de município em 1981, Sarandi enfrentou inúmeros problemas de infra-estrutura com implantação de loteamentos em forma desordenada, ocupados também pela população vinda da zona rural a procura de lotes com preços menores ${ }^{(10)}$. A economia do município está baseada, principalmente, na prestação de serviços e fornecimento da força de trabalho à cidade de Maringá, que é pólo industrial e comercial da região, atribuindo a Sarandi o título de cidade dormitório. Tal situação, do ponto de vista social resulta em baixa arrecadação para o município, pois os assalariados residentes também consomem em Maringá, afetando de forma importante o seu desenvolvimento em áreas como saúde, educação, habitação, assistência social entre outras ${ }^{(10)}$. Sarandi contava, em 2003, com aproximadamente 80.000 habitantes, o segundo maior índice de crescimento populacional do Estado ( $6,8 \%$ ao ano). O coeficiente de mortalidade infantil era de 15,7 óbitos por 1000 nascidos vivos em 2000, diminuindo para 12,9 em 2004, maior que o do município de Maringá que foi de 12,4 e 9,4 óbitos por 1000 nascidos vivos, nos dois anos, respectivamente. Existem no município 7 UBS sendo que 5 delas oferecem serviços de vacinação que funcionam de segunda a sexta-feira, de 7:00 às 17:00 horas. A cobertura do Programa de Agentes Comunitários de Saúde (PACS) é de $52 \%$ e entre as várias atividades desenvolvidas pelos 64 Agentes Comunitários de Saúde (ACS) estão incluídas as visitas domiciliares em sua área para verificar o estado vacinal de cada criança e fazer as orientações necessárias. A convocação dos faltosos ao programa de vacinação é feita por meio de aerogramas, telefonemas ou busca ativa.

O inquérito foi realizado em todo o município de Sarandi. Para realizar o inquérito nas regiões sem cobertura do PACS foi elaborado um roteiro de visitas. A coleta de dados foi feita por ACS devidamente treinados pelo setor de Vigilância Epidemiológica da Secretaria Municipal de Saúde, que utilizaram somente a carteira de vacina como fonte de informação. Nos casos em que a carteira de vacina não estava disponível ou na ausência dos moradores, várias visitas foram feitas ao mesmo domicílio. Mesmo com esses cuidados foram pesquisadas 740 carteiras vacinais o que representou $50 \%$ das crianças com até 23 meses de idade residentes no município, já que a estimativa do IBGE, para 2003, era de 1.482 crianças nessa faixa de idade. Os dados da carteira de vacinação foram 
transcritos em formulário próprio elaborado para a pesquisa. Quando o ACS encontrava alguma situação de atraso em alguma dose de vacina era realizada convocação imediata aos pais ou responsáveis pela criança para atualização da situação vacinal.

O esquema básico preconizado no Estado do Paraná compreendia, em 2003, as vacinas BCG, com 1 dose ao nascer; a Poliomielite, com 3 doses, sendo a primeira aos 2 meses e intervalo entre as doses de 2 meses; DPT e Hib, ou Tetra com 3 doses, sendo a primeira aos 2 meses e intervalo entre as doses de 2 meses; Hepatite $B$, com 3 doses, sendo a primeira ao nascer, a segunda, 30 dias após a primeira e a terceira dose 6 meses após a primeira; a VTV com uma dose e a FA também com uma dose, ambas aos 12 meses. Embora a idade mínima para conclusão do esquema básico seja de 12 meses, foi considerada com esquema básico completo, a criança que houvesse recebido todas as doses das vacinas antes de completar 24 meses de idade, independente da idade em que foram aplicadas e dos intervalos entre as doses. No caso da vacina Tetravalente o esquema foi considerado completo se a criança houvesse recebido as doses de DPT e Hib separadamente, ou todas as doses da vacina Tetravalente. Assim, o esquema completo foi a somatória das doses de DPT e Hib e as doses de Tetravalente. A cobertura vacinal foi analisada segundo sexo, faixa etária, tipo de vacina e município de aplicação da vacina. Os dados foram digitados e analisados utilizando o Programa EPI INFO 6.0. O projeto de pesquisa foi aprovado pelo Comitê Permanente de Ética em Pesquisa Envolvendo Seres Humanos da Universidade Estadual de Maringá (COPEP-UEM, parecer no 103/2004).

\section{RESULTADOS E DI SCUSSÃO}

Das 740 crianças de 12 a 23 meses cujas carteiras vacinais foram analisadas observou-se que $77,6 \%$ estavam com o esquema vacinal completo. Não houve diferença da cobertura vacinal entre meninos e meninas (Tabela 1 ). Em relação à faixa de idade observou-se que 68,9\% das crianças com 12 meses de idade ainda não tinham completado o esquema no momento da entrevista e para as crianças de 13 a 23 meses a cobertura vacinal foi de $81,7 \%$, mostrando RR para crianças com 12 meses de 3,77 ( $p<0,001)$ (Tabela 1).

Tabela 1: Cobertura vacinal em crianças de 12 a 23 meses de idade, segundo sexo e faixa de idade. Sarandi-PR, 2004.

\begin{tabular}{|c|c|c|c|c|c|c|c|c|}
\hline & \multicolumn{8}{|c|}{ Esquema Básico } \\
\hline & \multicolumn{2}{|c|}{ Completo } & \multicolumn{2}{|c|}{ I ncompleto } & Total & $\mathbf{R R}$ & IC (95\%) & $\mathbf{p}$ \\
\hline Sexo & & & & & & & & 0,810 \\
\hline Masculino & 319 & 77,2 & 94 & 22,8 & 413 & 1,03 & $0,79-1,35$ & \\
\hline Feminino & 255 & 78,0 & 72 & 22,0 & 327 & 1 & - & \\
\hline Faixa etária & & & & & & & & $<0,001$ \\
\hline 12 meses & 19 & 31,1 & 42 & 68,9 & 61 & 3,77 & $2,99-4,75$ & \\
\hline $13-23$ meses & 555 & 81,7 & 124 & 18,3 & 679 & 1 & - & \\
\hline
\end{tabular}

Quando a cobertura vacinal foi analisada para cada tipo de vacina observaram-se percentuais maiores para as vacinas que são administradas nos primeiros 6 meses de idade. A BCG apresentou a maior cobertura vacinal $(99,6 \%)$, seguida pela DTP/Tetra (98\%), Pólio $(97,2 \%)$ e Hepatite B $(96,2 \%)$. As vacinas, FA e VTV, que tem sua aplicação ao término do esquema básico, ou seja, aos doze meses de idade, apresentaram a menor cobertura, com valores de $85,7 \%$ e $85,8 \%$ respectivamente (Gráfico 1 ). Isto demonstra que o esquema vacinal das crianças analisadas está incompleto, principalmente, pela falta das últimas vacinas do esquema básico. 
Gráfico 1: Cobertura vacinal, em crianças de 12 a 23 meses de idade segundo tipo de vacina. Sarandi-PR, 2004.

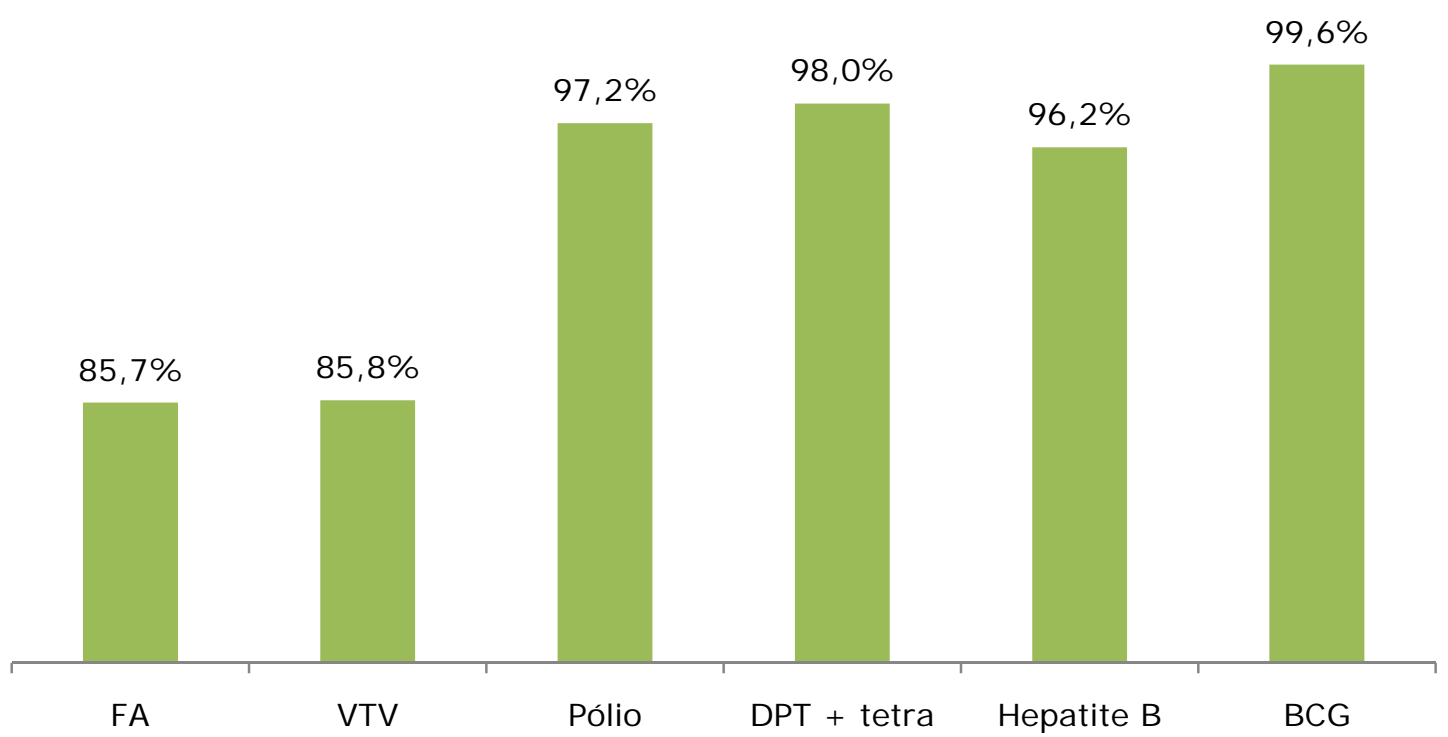

É importante observar que a cobertura para cada vacina específica mesmo sendo alta, não corresponde à cobertura do esquema básico, para o conjunto das vacinas, que foi de $77,6 \%$. Essa diferença entre cobertura individual das vacinas e cobertura para o esquema completo, do ponto de vista administrativo, significa que parcela das crianças de 12 a 23 meses residentes em Sarandi não está recebendo todas as vacinas preconizadas nas idades adequadas e nos intervalos corretos, apenas $3,3 \%$ das crianças vacinadas tinham completado o esquema na idade correta, com 12 meses de idade. Ao comparar esses resultados com os de pesquisa realizada em dois municípios do Estado da Bahia ${ }^{(11)}$ no ano de 1997, foi observada situação semelhante. Tanto em Sarandi como naqueles municípios baianos as crianças estão completando o esquema vacinal tardiamente.

Como comentado, a falta de manutenção de altas coberturas da vacina contra o sarampo (acima de $95 \%$ ) nos municípios brasileiros possibilitou novos surtos em $1997^{(9)}$. A meta de $95 \%$ preconizada para a VTV não está sendo alcançada, mesmo com as campanhas de vacinação contra a poliomielite que ocorrem 2 vezes por ano em todo o país, momento que também é utilizado para atualização do esquema vacinal das outras vacinas do programa. Em Sarandi a cobertura vacinal aos 23 meses de idade para a VTV, contra o sarampo, caxumba e rubéola, foi de $85,8 \%$, fato que deixa um número importante de crianças suscetíveis.

Observou-se diferenças nas coberturas das vacinas Pólio, DTP/Tetra e Hepatite $B$, considerando que o esquema básico, para as três vacinas, completam-se aos seis meses de idade. Mesmo que a diferença na cobertura seja pequena, esse fato pode sugerir de alguma forma, falhas de registro nas salas de vacina já apontadas em comparações realizadas entre dados de inquéritos de cobertura vacinal e dados administrativos, ou ainda a não realização das vacinas nos intervalos corretos. Essa diferença remete também a possíveis dificuldades dos serviços e usuários em adequar a aplicação da última dose da vacina contra Hepatite $B$ aos seis meses de idade juntamente com as últimas doses da Pólio e DTP/Tetra. O esquema da vacina contra a Hepatite $B$ é diferente, pois a $2^{\underline{a}}$ dose deve ser feita com 1 mês de idade e havendo atraso na aplicação da 2a dose o esquema já não será completado aos seis meses juntamente com a Pólio e DTP/Tetra. O grau de estruturação do programa de imunização interfere na cobertura vacinal, junto a outros dois condicionantes: a política de saúde e características da população ${ }^{(6)}$.

Com a descentralização dos serviços de saúde a partir de 1990 (Lei no 8.080, de 19 de setembro de 1990) houve a ampliação das responsabilidades municipais relativas à atenção à saúde. A descentralização juntamente com a utilização das campanhas nacionais de vacinação para aplicar as outras vacinas do calendário contribuiu para o aumento do impacto na melhoria das taxas de cobertura vacinal alcançadas nos últimos anos. A implantação do Programa de Saúde da Família (PSF) em 1994, incorporando o Programa de Agentes Comunitários de Saúde (PACS) com a reorganização das UBS, intensificou a resolutividade, com estabelecimento de vínculos de compromisso e responsabilidade entre os profissionais de saúde e população ${ }^{(12)}$. 
Para o Ministério da Saúde a taxa de abandono do programa de vacinação, que expressa o percentual de crianças que não chegou a completar o número mínimo de doses necessário para se proteger, tem como fatores mais comuns a falta de esclarecimento, a presença de reações adversas maiores do que as esperadas e o mau atendimento nos serviços de saúde ${ }^{(13)}$. Portanto, as causas do atraso na realização das vacinas nos intervalos corretos ou até mesmo a não realização das vacinas, devem ser conhecidas pelo serviço de saúde para que a equipe de trabalhadores, principalmente a equipe de enfermagem tome as devidas providências. Quanto ao município de Sarandi não foi investigado neste estudo o motivo das crianças não estarem com esquema de vacinação completo. No entanto, algumas razões para o não alcance das metas podem ser observadas, destacando-se a migração de famílias de outros estados e até de outros países cujas crianças não possuem o calendário vacinal compatível com o esquema utilizado no Estado do Paraná, o que caracteriza esquema vacinal incompleto. O esquema de vacinação em outros países é diferente do esquema brasileiro que oferece maior número de vacinas, caracterizando então crianças sem esquema vacinal completo. Outra possibilidade do não alcance da cobertura em Sarandi pode ser a evasão, vacinação das crianças residentes em outros municípios vizinhos, situação que acarreta menor cobertura vacinal administrativa, que é estimada pelo número de vacinas realizadas em relação ao número de crianças residentes.

A Tabela 2 mostra que crianças foram vacinadas em outros municípios, sendo a maior evasão encontrada para a BCG, DTP/Tetra e Pólio (13,9\%, $11 \%$ e $10,9 \%$ respectivamente). Para as vacinas VTV e FA a evasão foi menor $(4,3 \%$ e $5,4 \%$, respectivamente) e também menor taxa de cobertura vacinal (85,8\% e 85,6\%, respectivamente) (Gráfico 1 e Tabela 2). Visto que a cobertura vacinal é um indicador de acesso ao Programa Nacional de Imunização e de efetividade do programa local de imunização( ${ }^{(6)}$, tal fato alerta as equipes dos serviços de saúde do município a fatores, horário e qualidade do atendimento nas salas de vacina, os quais podem interferir no acesso ao programa local.

Tabela 2: Distribuição dos tipos de vacina segundo município da aplicação da última dose. Sarandi-PR, 2004.

\begin{tabular}{|c|c|c|c|c|c|c|c|c|c|c|}
\hline \multirow{4}{*}{ Vacinas } & \multicolumn{10}{|c|}{ Município de Vacinação } \\
\hline & \multicolumn{2}{|c|}{ Sarandi } & \multicolumn{6}{|c|}{ Fora de Sarandi } & & \\
\hline & \multirow{2}{*}{ no } & \multirow{2}{*}{$\%$} & \multicolumn{2}{|c|}{ Maringá } & \multicolumn{2}{|c|}{ Outros } & \multicolumn{2}{|c|}{ Sub-total* } & \multicolumn{2}{|c|}{ Total } \\
\hline & & & no & $\%$ & no & $\%$ & no & $\%$ & no & $\%$ \\
\hline BCG & 544 & 86,1 & 41 & 6,5 & 47 & 7,4 & 88 & 13,9 & 632 & 100 \\
\hline Hep B & 546 & 92,1 & 20 & 3,4 & 27 & 4,6 & 47 & 7,9 & 593 & 100 \\
\hline DPT+Hib e Tetra & 557 & 89,0 & 29 & 4,6 & 40 & 6,4 & 69 & 11,0 & 626 & 100 \\
\hline Pólio & 538 & 89,1 & 28 & 4,6 & 38 & 6,3 & 66 & 10,9 & 604 & 100 \\
\hline VTV & 516 & 95,7 & 13 & 2,4 & 10 & 1,9 & 23 & 4,3 & 539 & 100 \\
\hline FA & 505 & 94,6 & 16 & 3,0 & 13 & 2,4 & 29 & 5,4 & 534 & 100 \\
\hline
\end{tabular}

*Total de vacinados fora de Sarandi-PR.

Entre os outros municípios, Maringá foi o mais procurado para realização das vacinas, situação facilmente compreendida já que os limites entre os dois municípios é representado, em algumas regiões, por uma rua apenas. Além disso, grande parte dos moradores de Sarandi trabalha em Maringá, facilitando a realização de consultas, vacinas e outros atendimentos nos serviços de saúde de Maringá. Tal situação remete à discussão sobre o acesso aos serviços de saúde em geral e especialmente o serviço de vacinação. Para intervir nas causas do atraso vacinal e na evasão é indispensável que as vacinas estejam disponíveis não apenas em algumas, mas em todas as UBS e em período integral, além da ampliação do número de UBS de fácil acesso à população em todas as regiões do município. Com a instalação de Equipes de Saúde da Família (ESF) o acompanhamento do estado vacinal deve ser realizado com maior periodicidade, além do monitoramento das metas que deve ser estimulado e incorporado às atribuições das equipes de saúde.
Em relação às avaliações das coberturas vacinais das comunidades seria desejável que os inquéritos fossem realizados principalmente em anos distantes da realização dos censos populacionais proporcionando informações mais confiáveis já que em municípios menores existe dificuldade na estimativa populacional ${ }^{(2)}$. Por outro lado, é imprescindível que as equipes de saúde aprimorem a qualidade do atendimento realizado nas salas de vacina nas UBS, com anotações fiéis e cuidado com os relatórios e registros diários. Algumas iniciativas têm sido divulgadas para melhorar o processo de trabalho nos serviços de vacinação como nos municípios de São Carlos ${ }^{(14)}$, Ribeirão Preto(15), Santa Rosa (RS) ${ }^{(16)}$ e nos estados de Alagoas, Pernambuco e Sergipe ${ }^{(17)}$. A existência de um programa nacional que dê conta das diferenças locais parece ser a forma mais associada a melhores coberturas vacinais ${ }^{(6)}$.

$O$ registro efetivo das vacinas realizadas nas salas de vacina das UBS consiste em estratégia 
importante, fornece informações fidedignas acerca das doses aplicadas, contribui para o alcance das metas e permite realizar estimativa de retorno das crianças para as próximas doses. As atividades de educação em saúde proporcionam maior conhecimento da população acerca da importância da vacinação e provocam maior envolvimento e compromisso da equipe de saúde na realização desta atividade. A visita rotineira de ACS em todas as residências da área de abrangência consiste em atividade importante para monitorar a situação de saúde das crianças, realizando as orientações de acordo com cada caso, intensificando desta forma o aumento da cobertura vacinal e diminuindo os atrasos para completar o esquema básico.

É importante estar atento para os objetivos de erradicação e de controle de doenças que sejam passíveis de controlar e erradicar por meio da vacina, e que nas campanhas nacionais essa mentalidade seja intensificada. Entretanto a relevância das campanhas nacionais tem o inconveniente de passar a impressão de que a vacinação de rotina, do dia-adia não merece maiores cuidados. A vacinação de rotina não pode ser relegada em segundo plano, pois é uma ação excepcional, ainda mais agora que o PNI é um programa para toda a população e não apenas para as crianças. O atendimento de rotina não deve ser preterido, pois todo o dia é dia de vacina(1).

\section{CONCLUSÃO}

Os achados da presente investigação trazem importantes informações em relação à cobertura vacinal e às atividades de vacinação para os serviços de saúde mesmo considerando que $50 \%$ das carteiras vacinais de crianças do município foram pesquisadas. Os resultados do inquérito domiciliar mostraram índices satisfatórios de cobertura por vacina, para a BCG, Hepatite $B$, Anti pólio e DTP/Tetra. Já para as vacinas anti-sarampo (VTV) e febre amarela (FA) as coberturas foram abaixo do preconizado que é de $100 \%$. O conhecimento da real cobertura vacinal em Sarandi evidencia a necessidade de novos estudos com objetivo de identificar os motivos do atraso em completar o esquema básico. A detecção de evasão para vacinação em outros municípios sugere dificuldades de acesso e necessidade de atenção à oferta e qualidade dos serviços prestados.

A análise da situação vacinal efetuada com informações coletadas por meio de visitas domiciliares dos ACS no município de Sarandi, domicílio por domicílio, traz dados que além de ajudar a conhecer melhor os indicadores de saúde da população, remete a questões relativas às relações dos profissionais de saúde com os usuários. Uma das principais premissas da estratégia do PSF é estabelecer o vínculo, proporcionando maior proximidade dos profissionais com a comunidade. Então, além da validade técnica dos inquéritos domiciliares para levantar situação específica de saúde de uma determinada comunidade, as visitas domiciliares programadas e supervisionadas, possibilitam aprofundar o vínculo e possibilitam uma maior proximidade do trabalhador de saúde para conhecer o outro, já que, normalmente o atendimento se resume apenas a uma relação meramente técnico-assistencial, vendo o outro apenas como o excluído, o faltoso ou o não esclarecido e não cooperativo. As estratégias dos inquéritos domiciliares vêm então acrescentar elementos para, implementar os princípios do SUS, como universalidade e equidade, melhorando e aprimorando o vínculo e disseminando informações essenciais para melhorar a saúde da comunidade.

\section{REFERÊNCI AS}

1. Ministério da Saúde. Secretaria de Vigilância em Saúde. Programa Nacional de Imunização. 30 anos. Brasília: Ministério da Saúde; 2003. 208p.

2. Moraes JC, Ribeiro MCSA, Simões O, Castro PC, Barata RB. Qual é a cobertura vacinal real? Epidemiol. Serv. Saúde. 2003; 12(3): 147-53.

3. Moraes JC, Barata RCB, Ribeiro MCSA, Castro PC. Cobertura vacinal no primeiro ano de vida em quatro cidades do Estado de São Paulo, Brasil. Rev. Panam. Salud Públ. 2000; 8(5): 332-41.

4. Faversani MCSS, Kupek E, Westrupp MHB. Perfil epidemiológico do sarampo no Estado de Santa Catarina, Brasil, de 1996 a 2000. Cad. Saúde Pública. 2005;21(2): 535-44.

5. Fortes MRS. Enfermagem na Promoção dos Cuidados Primários na Saúde Pública. São Paulo: Livraria Everest Editora; 2002.

6. Moraes JC, Ribeiro MCSA. Desigualdades sociais e cobertura vacinal: uso de inquéritos domiciliares. Rev. bras. epidemiol. 2008; 11 Suppl1:S113-24.

7. Mota E. Inquérito domiciliar de cobertura vacinal: a perspectiva do estudo das desigualdades sociais no acesso à imunização básica infantil. Rev. bras. epidemiol. 2008; 11 Suppl 1:S125-8.

8. Luz PM, Codeço $C T$, Werneck GL. A reemergência da coqueluche em países desenvolvidos: um problema também para o Brasil? Cad. Saúde Pública. 2003; 19(4): 1209-13.

9. Carmo EH, Lunae, Dominguez CMAS. Situação da prevenção e controle das doenças transmissíveis no Brasil. In: Eduardo Hage Carmo; Expedito Luna; Carla Magda Allan S. Dominguez. (Org.). Saúde Brasil 2004: Análise da situação de saúde. 1st ed. Brasília: Editora Ministério da Saúde; 2004. p. 299399.

10. Prefeitura Municipal de Sarandi. [Internet]. Sarandi: Prefeitura Municipal (BR) [cited 2009 may 
25].

Available

from:

http://www. sarandi.pr.gov.br/home/index.php.

11. Porto LA. Cobertura vacinal nos municípios de Iguaí e Caldeirão Grande, Bahia, em 1997. Inf. Epidemiol. Sus. 1998; 7(4): 7-24.

12. Santos DM, Dubeux LS, Frias PG, Vanderlei LCM, Vidal AS. Avaliação normativa da ação programática Imunização nas equipes de saúde da família do Município de Olinda, Estado de Pernambuco, Brasil, em 2003. Epidemiol. Serv. Saúde. 2006; 15(3):2935.

13. Ministério da Saúde. Manual de Procedimentos Para Vacinação. 4th ed. Brasília: Ministério da Saúde; 2001. 316p.

14. Pedrazzani ES, Cordeiro AMA, Furquim EC, Souza FF. Implantação de um banco de dados em vacinação: experiência desenvolvida em um projeto de integração. Rev Latino-am Enfermagem. 2002; 10(6): 831-6.

15. Malta RF, Mishima SM, Almeida MCP, Pereira MJ B. $A$ utilização do inquérito domiciliar como instrumento de acompanhamento de ações de saúde em microáreas: analisando a situação vacinal de menores de um ano. Rev Latino-am Enfermagem. 2002; 10(1):28-33.

16. Balke EMR, Marchionatti CRE, Assunção MC. Cobertura vacinal contra o sarampo: inquérito vacinal de Santa Rosa (RS). In: Anais da 3ạ EXPOEPI. Mostra nacional de experiências bem-sucedidas em epidemiologia, prevenção e controle de doenças; 2003 nov 18-21; Salvador, Brasil. p. 58-9.

17. Cajueiro G, Almeida F, Nóbrega V, Farias A, Ferreira I. Monitoramento das coberturas vacinais contra o sarampo em crianças de 1 a 4 anos, nos municípios limítrofes entre os estados de Alagoas, Pernambuco e Sergipe. In: Anais da 3a Mostra nacional de experiências bem-sucedidas em epidemiologia, prevenção e controle de doenças; 2003 nov 18-21; Salvador, Brasil.p. 63-5.

Artigo recebido em 11.03.08.

Aprovado para publicação em 25.05.09. 